\title{
Lapurdum
}

Euskal ikerketen aldizkaria | Revue d'études basques |

Revista de estudios vascos | Basque studies review

$17 \mid 2013$

Numéro XVII

\section{Double sens, ironie, allusion, sous-entendu et insinuation dans le bertsularisme contemporain}

\section{Eric Dicharry}

\section{OpenEdition}

\section{Journals}

Édition électronique

URL : https://journals.openedition.org/lapurdum/2409

DOI : 10.4000/lapurdum.2409

ISSN : 1965-0655

Éditeur

IKER

Édition imprimée

Date de publication : 1 octobre 2013

Pagination : $37-53$

ISBN : 978-2-86781-409-9

ISSN : 1273-3830

Référence électronique

Eric Dicharry, « Double sens, ironie, allusion, sous-entendu et insinuation dans le bertsularisme contemporain », Lapurdum [En ligne], 17| 2013, mis en ligne le 15 novembre 2015, consulté le 22 juillet 2021. URL : http://journals.openedition.org/lapurdum/2409; DOI : https://doi.org/10.4000/lapurdum. 2409

Dicharry E. | IKER 


\section{Double sens, ironie, allusion, sous-entendu et insinuation dans le bertsularisme contemporain}

Eric DICHARRY

Cette recherche a pu être réalisée grâce à une bourse de recherche de la Fondation José Miguel de Barandiaran. Qu'elle en soit ici remerciée.

Nous tenterons ici de répondre à la double question suivante : comment se peut-il qu'un bertsulari dise une chose, veuille bien dire cette chose, et veuille dire aussi quelque chose d'autre? Comment est-il possible que l'auditoire comprenne, ce que Searle qualifie d'acte de langage indirect, alors que ce qu'il entend signifie autre chose?

Pour l'analyse du rire, la distinction type/occurrence est un instrument d'analyse précieux qui permet aussi bien la détermination du sens que de la vérité. Et c'est parce que la pragmatique s'intéresse spécifiquement à tout ce qui se rapporte aux rapports entre signes et locuteurs qu'elle nous est précieuse.

Pour faciliter la compréhension nous prendrons pour A, le locuteur, c'est-à-dire le bertsulari et pour B son auditoire. Lorsque A pense à X mais dit $\mathrm{Y}, \mathrm{B}$ écoute $\mathrm{Y}$ mais doit comprendre $\mathrm{X}$ pour en rire. Si A parvient à faire penser à X alors qu'il dit $\mathrm{Y}$, c'est qu'une connivence entre A et B s'est instaurée. C'est sur cette proximité-intimité que le rire peut s'épanouir et s'émanciper. Le bertsulari pour faire rire joue sur les occurrences d'un même signe. Il manipule les occurrences, nommées token dans la terminologie de Charles Sanders Pierce et par les chercheurs anglais et américains qui lui ont succédé, token, d'un même type, ce dernier étant considéré comme "l'entité idéale, toujours identique à travers ses réalisations diverses ». (Armengaud, 1999 : 21) Le corpus nous a gratifié avec hontza, d'un type, et de deux occurrences, hontza=imbécile, hontza =hibou. Mais le bertsulari (A), n'énonce pas les deux occurrences dans son discours. Il se contente d'en énoncé une, en espérant que les auditeurs (B) seront dans la capacité de remonter le cheminement de sa pensée pour accéder à la seconde occurrence, non dite. Rire, c'est donc d'abord, et avant tout, maîtriser les conventions des signes du langage et des codes culturels, les définitions et les jugements. Lallocutaire doit être capable de saisir comment le locuteur se sert du mot et ce qu'il en fait. Avant d'être susceptible d'en rire, il doit être d'abord, au préalable, dans la capacité de saisir ce dont il parle et comment il en parle pour parvenir ensuite à capter comment il le comprend et enfin accéder par le vocabulaire et par la grammaire aux «transactions linguistiques réelles » (Wittgenstein, 1980 : 44). Ce partage partiel des signes par une maîtrise aléatoire entrainerait 
une réduction drastique des occasions de rire. La théorie Piercienne triadique et dynamique de la sémiose rend compte que «le signe est une relation conjointe avec la chose dénotée et avec l'esprit ». (Peirce, 1978 : 143) Cette définition du signe permet de conclure que pour que le rire se fasse audible il est impératif que les interprétants (à ne pas confondre avec les interprètes) du signe expriment la même relation du representatem et de l'objet. Le mot hontza est intimement lié à l'usage qu'en font locuteur et allocutaires. Hors de ce partage de l'interprétant et du sens du mot entre le bertsulari et ses allocutaires, point de rire. Le rire est un domaine réservé à ceux qui possèdent des interprétants identiques.

Nous pouvons proposer le schéma suivant pour résumer notre pensée :

$\begin{array}{lccc}\begin{array}{l}\text { (Pensée non énoncée par) A } \\ \text { (Interprétation de X en Y, }\end{array} & \text { X } & \text { Y } & \begin{array}{l}\text { B (Enoncé capté puis interprété) } \\ \text { (Réinterprétation Y=X=RIRE) }\end{array} \\ \begin{array}{l}\text { Rité intérieur) } \\ \text { Dit/énoncé par A }\end{array} & \text { Y } & \text { X } & \text { Pensée de A retrouvée par B }\end{array}$

C'est au moment de la réinterprétation de $\mathrm{Y}$ en $\mathrm{X}$ que le rire se fait audible chez l'auditoire. Il ne sera généralisé que dans la mesure où l'intégralité des allocutaires auront réussi à voir en $\mathrm{Y}$ une part de $\mathrm{X}$. Si B est incapable de remonter à X, il se prive du rire. Dans l'exemple de hontza, si le public rit, c'est qu'il a effectué le passage de la première occurrence, hontza=hibou, à la seconde, hontza=imbécile. Cheminement qui n'a pas eu besoin de changer de type (hontza). Le bertsulari profère un bertsu qui veut dire exactement ce qu'il dit, et veut dire aussi quelque chose d'autre. Le type n'est pas dans ce cas précis la source du rire. C'est bien plus ici le jeu sur ses occurrences qui initie le rire. Avant d'en rire les allocutaires ont dû se poser la question suivante :

\section{«Est-ce que ça (hontza) signifie quelque chose?» (1)}

Hontza symbolise le départ d'une relation signifiante marquée par signifie quelque chose et se classe parmi les « choses signifiantes ». Les allocutaires à l'aide de "relateurs » tels que signifie, veut dire ou est, c'est répondent à la question (1) et formulent une double réponse. Hontza $\mathrm{H}$, signifie à la fois $\mathrm{H} 1$, hibou et $\mathrm{H} 2$, imbécile. Cette mise en relation entre $\mathrm{H}$, d'une part et $\mathrm{H} 1$ et $\mathrm{H} 2$ d'autre part, exige que $\mathrm{H} 1$ et $\mathrm{H} 2$ soient compréhensibles pour les allocutaires de manière à ce qu'ils puissent tester les deux significations de hontza pour en dernier lieu en choisir une. C'est dans l'élection de $\mathrm{H} 2$ plutôt que de $\mathrm{H} 1$ et dans la connaissance du double sens ( $\mathrm{H} 1$ et $\mathrm{H} 2$ ) de $\mathrm{H}$ que le rire trouve sa racine. Seuls les allocutaires connaissant la polysémie $(\mathrm{H} 1+\mathrm{H} 2)$ de hontza peuvent en rire. Ceux qui ne comprennent que $\mathrm{H} 1$ dans $\mathrm{H}$ restent insensibles au comique initié par la polysémie de hontza.

Le passage de X à Y n'a pas obligatoirement à changer de type pour être opérationnel. Ainsi avons pu au fil des textes repérer les translations et glissements qui suivent:

Seaska (nom propre de l'association) - seaska (nom commun, berceau) gibela (nom commun, foie) - gibela (substantif, l'arrière) Leon (nom propre de la province) - Leon (nom propre du prénom masculin) L.A.B ekoa (sigle) - labekoa (nom commun, four) 
poto egin (vocabulaire du bertsularisme)- poto egin (cagnotte)

ofizio (nom commun, métier, profession)- ofizio (nom commun, travail, vocabulaire du bertsularisme)

txapela (nom commun, béret) - txapela (nom commun, vocabulaire du bertsularisme) hontza (nom commun, le hibou)- hontza (imbécile, idiot)

piper-ik (piment) - piperrik ulertu (ne rien comprendre)

De plus, si modification de type il y a, ce peut être pour un type qui par ses similitudes renverra au premier type ce qui permettra au public de remonter à la source.

Castilla Leon (nom propre de la province) - Maurizia ta Leon (noms propres, prénoms)

Patxi Lopez (nom propre, prénom basque) - Paco Lopez (nom propre, prénom espagnol)

Patxi Lopez (nom propre, prénom basque) - Franco Lopez (nom propre, prénom du nom de l'ancien dictateur)

Zazpi Etxe Franzian (nom d'un ouvrage) - Zazpi etxe dauzka han (sens commun avec allusion au titre)

zakurrak (sens commun, les chiens) - txakurrak (sens figuré, les forces de l'ordre, les policiers)

Me kauen la puta - Me kauen la fruta

Le rire se situe au niveau de la manipulation des unités sémantiques suivantes : le mot, le syntagme (deux mots ou plus), le vers (ou phrase), le bertsu, le discours qui intègre l'ensemble des bertsu. Il privilégie les « unités de première articulation » pour reprendre la description d'A. Martinet (1964 : 17), en s'intéressant majoritairement aux mots et/ou aux morphèmes (ou monèmes) plutôt qu'aux phrases et au discours dans son ensemble. Le jeu sur les unités de deuxième articulation, où l'on isole des unités minimales distinctives au seul niveau phonique: les phonèmes, joue un rôle subalterne quant il s'agit de faire rire. L'effet comique est généralement compris dans ce jeu sur le sens des mots qui est le terrain privilégié des bertsularis. Pour que le rire se fasse audible, il faut que le « double bouclage », du locuteur et de l'allocutaire, soit achevé. Il est indispensable que le sens soit partagé. Comme le note Irène Tamba-Mecz :

"Il faut on le sait, attendre la "fin» d'un mot, d'un syntagme ou d'une phrase pour pouvoir identifier le sens. Tant qu'un vocable n'est pas articulé jusqu'au bout, je ne sais pas de quelle "unité " il s'agit (...). Il en va de même pour un syntagme ou une phrase dont la grammaire détermine les possibilités de "bouclage » et le locuteur, le «bouclage " effectif. (...) Dans la langue, seuls les points de synthèse sont indiqués, non le résultat sémantique que l'on peut vérifier en le reformulant par une paraphrase explicitement donnée comme équivalente par des relateurs du type : c'est-à-dire, signifie. » (Tamba, 1998: 50)

Analysons maintenant le bertsu suivant :

Ai zure hitzen jasa / ezin da atertu

ta gustora nahi nauzu / txokoan baztertu. 
Baina hala ere naiz / hementxe agertu

barkatu nik ez dizut / piperrik ulertu. (bis)

(La bourrasque de tes mots ne peut s'interrompre / et tu aimerais me mettre de côté / mais cependant j'ai apparu ici (à Mauléon) / excuse moi mais je ne t'ai pas du tout compris.)

Le passage de piper-ik à piperrik ulertu est ici exemplaire. Ce n'est qu'à partir du moment où l'improvisateur aura terminé d'énoncé son bertsu, à la fin de son énonciation, à l'instant même où les allocutaires auront terminé le «bouclage prébouclé » par le locuteur que leur rire se fera audible. C'est ce qui permet d'expliquer pourquoi le rire se fait audible à la fin de l'énonciation et non pas avant. Car, ce n'est qu'après avoir répondu à la double question : «Qu'est-ce que signifie piperrik ulertu ? et «A quoi fait référence ce piperrik ulertu ? », et seulement à partir de ce moment précis, que les allocutaires peuvent profiter du sens comique de l'énoncé. C'est au moment où ils comprennent que le bertsulari joue sur le sens du mot " piperik», à l'instant même où Eneritz Zabaleta rebondit sur le piperrik déjà énoncé par son adversaire de la joute, en utilisant le syntagme « piperrik ulertu » que le public en rit. Pas avant. Il ne s'agit pas, ici, d'un méta rire, puisque le public n'avait pas ri à l'écoute de l'énoncé du premier piperrik. Le rire n'est pas, dans ce cadre, limité aux frontières d'un bertsu. Il naît de la reconnexion du bertsu présent à un autre bertsu qui le précède et sans lequel il n'existerait pas. Le rire transbertsu, fruit du résultat de l'association sémantique d'un mot, piperrik et d'un syntagme, piperrik ulertu, découle, chez les allocutaires, d'une mémoire auditive à double détente qui mêle mémoire auditive immédiate par le souvenir de piperrik ulertu et mémoire auditive antérieure par la remémoration de piperrik. Sans l'antérieure (plus ou moins éloignée de l'instant t marquant la fin de l'énonciation), l'immédiate serait non opérationnelle. Un spectateur qui se serait absenté lors de l'énoncé du premier piperrik serait dans l'incapacité de rire du second. Le rire transbertsu est conditionné par l'activation mnémonique à double détente.

Limprovisateur basque ne se prive pas de se servir des variations qui existent entre les divers usages des mots. Derrière les ressemblances phoniques se cachent des dissemblances de significations. Ces variations sémantiques, qui peuvent être fonction des localisations géographiques, sont repérées et mises à contribution pour la construction des bertsu. C'est par exemple le cas avec le mot gibela qui possède un sens spécifique au Pays Basque nord. Dans les trois provinces du nord, gibela est polysémique (il signifie « foie » et « derrière ») alors qu'au sud il est monosémique (il signifie seulement, le « foie »). Amets Arzallus joue sur cette variabilité pour faire rire. Mais le rire peut, de plus, se focaliser sur les sigles, sur les prénoms ou sur les noms, en changeant tout ou une partie de leur formulation. L.A.Bkoa devient labekoa et Patxi Lopez est transformé en Paco Lopez mais également en Franco Lopez. Dans ce cas le patronyme reste inchangé. C'est sur le prénom que sont effectués les variations et les changements qui vont conduire au rire. Lart du bertsulari consiste à rendre manifeste et compréhensible le sens de ces passages et de ses transformations. Pour faire rire, l'improvisateur fait mine de ne pas distinguer « la peau de la chemise » (Montaigne, Essais, III : 10) comme dans l'exemple de seaska, où le locuteur fait semblant de ne pas voir derrière le premier sens de ce mot (le berceau), le second (le nom de l'association des écoles basques). Pour parvenir à ses fins, il profite des « phénomènes de cumul (polysémie) ». (Tamba-Mecz, 1998 : 45) Les innovations se font sur le patron de configurations antérieures, comme dans 
l'exemple du passage de Me kauen la puta en Me kauen la fruta. Comme le notait Saussure, " La langue est une robe couverte de rapiéçages faits avec sa propre étoffe». (Saussure, CLG : 235)

Lobjectif est clair : faire rire en jouant sur la multiplicité du sens des mots, sur les ressemblances phoniques en même temps que sur les différences de sens. Le rire sémantique s'émancipe sur le « développement d’un système de sens seconds» (Barthes, 1985, p. 33), la connotation, qui «parasite » la langue proprement dite. Ainsi perçu, le rire est réaction au déplacement du sens quand un mot « est mis » pour un autre, quand le signe glisse d'un sens à un autre, comme c'est le cas dans la métaphore (Riffaterre, 1982 : 91), résultat du repérage d'un « parasitage » linguistique initié par un dédoublement de la langue. Le rire implique une reconnaissance collective de la connotation, de la polysémie, du dédoublement de la langue, du sens, des néologismes sémantiques (en percevant les nouvelles acceptions que les bertsulari donnent aux signes déjà existants) et d'emprunts (surtout dans les usages de la langue dite vulgaire, gros mots, injures...).

Si le passage de X à Y peut se faire par modification des occurrences, sans changer de type, il peut également revêtir d'autres formes en ayant recours aux images, aux figures de style et de pensée. Ces dernières, dont les métaphores et les métonymies font parties, peuvent être particulièrement utiles dans des conditions d'énonciation spécifiques comme par exemple à l'heure d'interdictions particulières. Si A décide de penser X, mais s'il ne peut le dire, il utilisera alors Y pour que le public saisisse le fond de sa pensée. De plus, il donnera assez d'indices à $\mathrm{B}$ pour qu'il puisse sans difficulté retrouver $\mathrm{X}$ dans $\mathrm{Y}$. Tout son art consistera à incorporer à son dit suffisamment d'opacité pour que son énoncé ne soit pas compris, par ceux à qui il refuse cet entendement. Le recours à ce travestissement de X en Y est multifonctionnel. Il permet au bertsulari de faire rire son auditoire mais aussi de contourner les interdits, de transgresser les conventions sans avoir à être inquiété. Car, en fin de compte, la responsabilité de la réinterprétation incombe à $\mathrm{B}$ et non à $\mathrm{A}$.

Prenons un exemple pour clarifier notre propos. Lorsque Sustrai Colina énonce :

ta gurean otsoak tiroz hiltzen dira

et chez nous les loups meurent à coup de fusil

Limprovisateur A n'évoque pas l'organisation séparatiste basque E.T.A dans son dit. C'est B, Karlos Aizpurua, qui interprète (interprétation recueillie par nos soins dans un entretien) que A a voulu signifier que cette métaphore renvoie à l'organisation. Pour être capable de comprendre le rire des bertsulari il est indispensable d'accéder à leur monde mental, à leur monde de significations qui ne peut se faire que grâce à une exhaustion attentive du vocabulaire bertsularistique tiré de l'énoncé de la performance. Cette étape préliminaire est nécessaire et cruciale à l'heure d'accéder au sens véritable. Derrière la concaténation bien faite se dégage une multitude de sens plausibles, ad infinitum que seul l'affinage, par l'entretien qui instaure une proximité avec le créateur, peut révéler et circonscrire. Il ne s'agit pas de proposer des sens qui dépasseraient ceux des créateurs mais de réduire la distance du signifiant au signifié par l'entretien avec les artistes du langage improvisé-chanté. L'important en fin de compte n'est pas tellement de savoir ce que le rirologue saisit. Ce qui prime c'est ce que le bertsulari a voulu que l'Autre saisisse et ce que l'Autre a saisi. C'est distinguer ce que l'improvisateur basque a refoulé, cerner ce refoulement en répondant à la question : pourquoi a-t-il refoulé ce qu'il a refoulé ? Toute la difficulté réside alors à faire dire au bertsulari justement et précisément ce qu'il 
ne voulait surtout pas dire et qu'il avait tout de même réussi à dire en usant de stratagèmes (métaphores, double sens, allusions...). Les repérages des déplacements, des surplus ou des peu-de-sens viennent tels des béquilles idéatives circonscrire l'objet au-delà du sens littéral. Le bertsulari peut trouver un énoncé adéquat, l'avoir sur le bout de la langue tout en ne le prononçant pas. C'est de cela qu'il est question ici, de repérage de déchet d'un mot mis à la place d'un autre, refoulé mais non oublié. Le rirologue se retrouve bien souvent en face d'un résidu, d'un reste, d'un remplacement, d'un peu de sens au sens Lacanien du terme, qui ne fait sens qu'une fois complété. En d'autres termes, si l'E.T.A est absent c'est que gurean est venu prendre sa place dans une métaphore réussie. Suivons Jacques Lacan dans sa définition:

"Nous retrouvons là la formule de la métaphore en tant qu'elle s'exerce par un mécanisme de substitution d'un signifiant Sà un autre signifiant S'. Quelle est la conséquence de cette substitution ? Il se produit au niveau de S' un changement de sens - le sens de S', disons s', devient le nouveau sens, que nous appellerons s, pour autant qu'il correspond à grand S. Pour ne pas laisser subsister d'ambiguité dans votre esprit, car vous pourriez croire que dans cette topologie petit s est le sens de grand $S$, je précise qu'il faut que le $S$ soit entré en relation avec S' pour que le petit s puisse produire, à ce titre seulement, ce que j'appellerai s". C'est la création de ce sens qui est la fin du fonctionnement de la métaphore. La métaphore est toujour réussie pour autant que cela étant exécuté, exactement comme dans une multiplication de fraction, les termes se simplifient et s'annulent. Le sens est alors réalisé, étant entré en fonction dans le sujet. » (Lacan, 1998 : 59-60)

Il faut entendre : ta E.T.A-k otsoak tiroz hiltzen ditu à la place de ta gurean otsoak tiroz hiltzen dira. A l'ethnolinguiste de savoir le remettre à sa juste place avec tout l'inconfort que la démétaphorisation suppose et engendre. Bien que le nom de l'organisation séparatiste ne soit que rarement mentionnée, l'E.T.A n'est cependant pas totalement évacuée des joutes oratoires. Il peut en être question sans que son nom soit prononcé. Pourtant le public saisit parfaitement de quoi il est question. Comme le note Joxerra Garzia qui revient sur une session ayant eu lieu à Zarautz au Pays Basque Sud :

"It seems that bertsolaris now feel able to deal with topics that were taboo just a few years ago. It is telling that it was, in fact, a bertsolari himself who dared to propose one of the latest deadly terrorist attacks perpetrated by ETA as a theme for improvisation. The bertsolari in question was Jon Sarasua, who was acting at the time as a theme-prompter rather than a performer. The session was being held in Zarauz, where one month earlier terrorists had killed Iruretagoiena, a local politician and member of the right-wing PP party. The exact theme proposed by Sarasua was : "Less than a month ago a man was killed here in Zarauz." The bertsolaris to whom this topic was addressed were Andoni Egaña and Jon Maia. »

Et il donne le bertsu d'Andoni Egaña que nous reprenons ici :

Hilabete bat oraintxe dala

nago pentsatzen hasia; holakoetan geratzen zaigun itxaropen eskasia! 


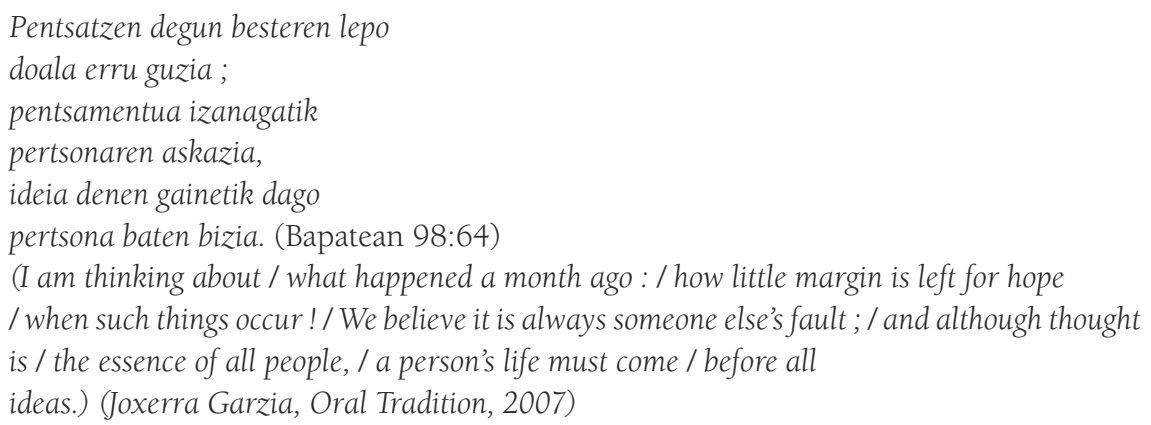

Dans notre exemple évoqué plus haut, Sustrai Colina échappe à toute suspicion d’apologie du terrorisme car en réalité ceux ne sont ni lui, ni Karlos Aizpurua qui énoncent les bertsu mais les personnages virtuels qu'ils incarnent, le berger et le géomètre. Tout pouvoir judiciaire, quel qu'il soit, est impotent face aux personnages fictifs du monde imaginaire à moins de pratiquer la censure du genre, censure pratiquée sous le joug de la dictature franquiste. Lidentité de l'auteur n'autorise pas de questionnement lorsque le bertsulari improvise pour son compte. Le performeur recouvre alors parfaitement l'auteur. La question mérite d'être posée dès qu'il intègre un rôle, un personnage. Est-ce dès lors le bertsulari qui s'exprime ou le personnage qu'il incarne? Quelle est la part de lui-même dans son dit ? Qui parle? Sustrai Colina ou le berger qu'il interprète ? Comme le confiait Domingo Arranbide à la journaliste Gaxuxa Arranbide lors de la célébration annuelle du Bertsulari Xalbador à Urepel les 10 et 11 octobre 2009:

«Badakizu, zure sentimendu edota pentsamenduetatik kanpo gezurretan ezin luzaz aritu. Holako bertso sentikorrak botatzeko barnetik etorritako hitzak besterik ezin dira izan. » (Domingo Arranbide, Kazeta.info, 2009ko urriaren 10a)

"Vous savez, le mensonge ne peut jamais s'émanciper longuement de votre sentiment ou de votre pensée. Pour improviser des bertsos sensibles, la seule manière possible c'est que les mots viennent de l'intérieur, de la profondeur de l'âme. » (Domingo Arranbide, Kazeta.info, 10 octobre 2009)

Lénoncé est personnel, propre à l'improvisateur, du fait même que le bertsulari est celui qui dit le texte, mais aussi et surtout, parce qu'il est celui qui le compose dans l'instant. Lauditoire ne peut nier son existence en tant que créateur du dit. En matière d'improvisation orale basque, se référer à l'auteur n'est point une «érudition de lettré ». (Zumthor, 1983 : 212) Linterprète n'est plus seulement «l'individu dont on perçoit, à la performance, par l'ouie et la vue, la voix et le geste. » (idem, p. 213) Il est aussi l'interprète qui interprète un rôle. Le bertsulari est à la fois lui-même (Sustrai Colina) et l'autre (le berger). Lincarnation du personnage brouille les cartes. Elle introduit un dédoublement de l'interprète qui instaure une double détention du sens. Elle a une fonction de cryptage similaire à celle que l'usage de la métaphore instaure en passant de $\mathrm{X}$ et $\mathrm{Y}$. Tous deux sont des procédés qui permettent aux bertsulari de mettre une distance entre eux et leur dit. Par le recours à ces stratagèmes qui cultivent l'ambiguité, ils s'affranchissent d'une censure politique et/ou morale et se servent de la poésie pour, tant 
bien que mal, parvenir à leurs propres fins. Ils peuvent ainsi dire, sans le dire, tout en le disant (en instaurant une distinction entre sens littéral et sens insinué), ou dire sans l'avoir personnellement dit (ce qui est dit étant énoncé dans le cadre de l'interprétation d'un rôle). Le rirologue se retrouve face à un double dédoublement de l'être et du dit. Rôles et figures de style sont les garants de la liberté de dire des bertsulari et les deux grands piliers du rire.

La performance, par une implosion de sens, refuse la privatisation du langage et derrière la pure musicalité de la voix, le texte impose sa force. En déclamant leur vérité, les improvisateurs basques échappent à la névrose. L'engagement politique des bertsulari militants, porte-voix de leur communauté, porte-parole du peuple, implique la nécessité de faire passer un message explicite (toujours compréhensible même s'il est codé) qui confère une importance telle aux paroles, au texte poétique, et aux mots, qu'ils retrouvent leur autonomie vis-à-vis de la musique. C'est le sens, initié par le texte poétique qui désaliène, plus que la forme et le son. C'est lui qui l'emporte sur l'attention des auditeurs. La musique n'y est presque pour rien, à la limite elle devient inaudible, simple prétexte au message.

La forme, une fois validée par le public, s'efface pour laisser la vigilance se concentrer sur le fond du texte poétique.

"Parole poétique, voix, mélodie - texte, énergie, forme sonore - activement unis en performance concourent à l'unicité du sens. (...) C'est au niveau du sens qu'est scellée l'union : le sens en constitue le gage. Le reste en découle.» (Zumthor, 1983 :184)

C'est l'influence de la vie réelle (situation politique, conflit, confrontations idéologiques) qui en dernière instance conditionne l'aptitude des exécutants comme de leurs allocutaires à percevoir une domination entre les deux arts (musique et texte poétique) dans l'improvisation et/ou à penser les rapports qu'ils entretiennent. Par temps de crise, il ne s'agit plus simplement d'improviser la poésie en la chantant, tout en respectant sa musicalité et ses règles de composition. Lobjectif nouveau est clair : charger le texte poétique de sens en l'orientant idéologiquement. Le temps de l'improvisation pour l'improvisation est révolu (tout du moins pour certains improvisateurs particulièrement politisés). Il est désormais impératif de critiquer une réalité pensée comme insupportable, d'improviser pour changer le monde (en riant ou en en riant pas, c'est fonction du contexte, du locuteur, des allocutaires, du thème abordé...).

Pour construire son interprétation, le poète de la voix va faire appel à ce que Hansson définit comme « la pragmatique du second degré » qui est «l'étude de la manière dont la proposition exprimée est reliée à la phrase prononcée, là où, dans les cas intéressants, la proposition exprimée doit être distinguée de la signification littérale de la langue». (Armengaud, 1999: 47) Karlos Aizpurua se réfère au contexte pour le second degré qui est « le contexte au sens élargi de Stalnaker, c'està-dire à ce qui est présumé par les interlocuteurs. C'est un contexte d'informations et de croyances partagées. Ce n'est pourtant pas un contexte "mental " mais un contexte traduit en termes de mondes possibles. » (idem, p. 47) Dans ces conditions A se dégage de toute responsabilité. Il fait entendre sans prendre la responsabilité d'en communiquer intentionnellement la teneur. On ne peut tenir A pour responsable qu'à travers son dire et son dit et non pour ses pensées. Si le bertsularisme est un espace de liberté, c'est à X qu'il le doit, bien plus qu'à Y. Mais cette liberté n'est pas illimitée. Elle est conditionnée voire subordonnée aux présuppositions de l'auditoire qui peuvent jouer un rôle majeur à l'heure d'orienter le sens des énoncés. Son dire et son dit sont, dans ces conditions, inféodés à ce que le public à envie d'entendre. Lorganisation 
du discours autour de l'allocutaire induit une modification de l'énoncé du locuteur. C'est le contexte de profération qui donne au texte sa couleur car il affecte à la fois la force avec laquelle la proposition est exprimée, et en même temps, la proposition elle-même. L'analyse du rire ne peut faire l'impasse d'une approche tridimensionnelle qui insiste synchroniquement sur les aspects syntaxiques, sémantiques (étude de la relation du langage aux objets) et pragmatiques (étude du langage et des personnes qui l'utilisent).

Le rire est compris dans cet interstice laissé volontairement vaquant par le bertsulari, entre X et Y, entre sens figuré ou métaphorique et sens littéral, entre sens ouvertement exprimé ou communiqué et sens insinué, entre ce qui est dit et ce qui est pensé. C'est dans cet interstice que se laisse appréhender l'ironie du bertsulari qui énonce l'inverse de ce qu'il pense.

"Quand tout est simple, le locuteur profère une phrase et il entend signifier par là exactement ce qu'il dit littéralement. Quand les choses se compliquent, le sens de la phrase comme telle, et le sens pris par le propos proféré par le locuteur, cessent de se recouvrir. On entre dans le monde sinon des sortilèges du moins dans un monde où les apparences se font piège: sous l'herbe verte glisse le serpent de l'ironie, de l'insinuation, de l'allusion, du sous-entendu de l'équivoque, du double sens. La politesse exquise et le persiflage sournois se donnent la main. » (Armengaud, op cit: 94)

C'est au moment où l'auditoire parvient à mettre à jour le fond de la pensée de l'improvisateur qu'il rit. Le rire s'inscrit dans la relation logique entre énoncés et sera activé par l'auditeur en reconnectant Y à X, en parcourant le chemin qui sépare (tout en les réunissant, réuni-sens) les différentes acceptions. C'est lors de ce cheminement que le rire nait. Pour prendre un exemple, c'est au moment où le public reconnecte le lit avec l'acte sexuel susceptible de s'y produire que l'auditoire rit. Les rires éclatent lorsque l'auditoire épuise l'insinuation en complétant totalement la suggestion. Ce n'est qu'à l'heure où l'« implicature », pour reprendre la terminologie de Grice, est assimilé dans sa substance que le rire se fait audible. C'est dans l'acte digestif instantané de mots et de pensées que l'auditoire trouve matière à en rire. Le rire est un épuisement, une assimilation par phagocytation des sens.

"Dans les actes de langage indirects le locuteur communique à l'auditeur davantage que ce qu'il dit effectivement en se reposant sur un arrière-plan d'informations linguistiques et non linguistiques, en même temps que sur la capacité d'inférence rationnelle de l'auditeur. L'appareil théorique requis pour expliquer la partie indirecte des actes de langage indirects consiste donc en: la théorie générale des actes de langage, des principes généraux de coopération conversationnelle (tels que Grice les a formulés) et un arrière-plan d’informations factuelles partagées par le locuteur et l'auditeur.» (Armengaud, op cit: 95)

Pour que le rire puisse s'émanciper il faut que le sous-entendu du bertsulari soit entendu par l'auditoire. La reconnexion est facilitée par une recontextualisation de l'énoncé dans un contexte qui détermine selon Schnelle «si un énoncé sérieux a été fait, plutôt qu’une plaisanterie ». (Schnelle, cité par Armengaud, p. 48) Le rire qui varie en fonction des circonstances de l'énonciation, des présuppositions de l'auditoire et du contexte de profération n'est pas obtenu sans effort. Il demande un travail de recomposition dans l'infiniment bref de l'instant. Il s'offre à l'auditeur au prix d'une figuration du littéral, d'une exégèse de la littéralité, d'un labeur neuronal, tel une récompense. Pour le bertsulari, il est le signe que son public l'a compris, 
qu'ensemble ils forment un nous (je+vous). Comme le note Françoise Armengaud, « pour sousentendre que q (que l'on ne veut pas dire ouvertement pour telle ou telle raison), il suffit de tenir un propos $p$-n'importe lequel fait l'affaire, pourvu que p implique conversationnellement q » (op cit, $p$. 70). Pour Grice, il y a sous-entendu lorsque l'auditeur peut faire le raisonnement suivant :

"Le locuteur a dit que p; il n'y a pas de raison de supposer qu'il ne respecte pas, dans la mesure du possible, les principes de la conversation; en l'occurrence, il ne les respecte dans la mesure du possible que si q; il sait (et sait que je sais qu'il sait) que je puis m'apercevoir que la supposition que q est requise pour continuer à présumer qu'il respecte les principes conversationnels; il n'a rien fait pour empêcher de penser q; il me laisse donc entendre que q » (Grice, 1975)

Pour mieux saisir le fonctionnement de l'implicature nous reprendrons ici l'exemple développé dans le chapitre 4 sur le rire et les allusions sexuelles. Beñat Iguaran :

Horrelakoak egiten dira / eta gainera askotan

sofan sarritan geratzen zera / descuido batean lotan

Eta zinean pelikula bat / txarra bada egon hortan

biok musuka hasi gintezken / zinek oso ta goxotan

(Des comme cela se font / et en plus souvent / tu restes souvent sur le sofa / endormi et inattentif. / Et au cinéma un film / s'il est mauvais pendant ce temps / nous pourrions commencer à nous embrasser / tout le film à l'aise.)

\section{Oier Lakuntza :}

Muxuka hasi ta pelikula / ia ia osorik galdu

ta hortarako ordaintzen ahal da / haren mesedez azaldu.

Muxutarako etxean ere / hobe egongo gara gu

sofan nekatzen balinbagara / ohea hurbil daukagu (bis)

(Les baisers commencés le film / sera presque entièrement raté / et est-ce pour cela que nous devrions payer / éclaire moi à ce sujet. / Pour des baisers, à la maison aussi/ nous serions mieux / si nous nous fatiguons sur le sofa / nous avons le lit à côté.)

C'est la théorie Gricéenne de la communication (pour laquelle tout échange conversationnel entre un locuteur et un destinataire suppose un minimum d'entente, un minimum d'effort coopératif) qui sera mis ici à contribution. L'échange entre les deux participants implique le respect de règles communes. Grice soutient que les participants engagés dans un échange sont censés observer un principe de coopération. Ce principe enjoint les participants à ce que leur contribution, au moment de l'échange, soit conforme à la direction et au but exigés par cet échange. Elle précise ce principe par quatre catégories de maximes dites « conversationnelles»:

«Maximes de quantité 1. Que votre contribution soit aussi informative que nécessaire. 2. Que votre contribution ne soit pas plus informative que nécessaire. Maximes de qualité 1. Ne dites pas ce que vous croyez être faux. 2. Ne dites pas les choses pour lesquelles vous manquez de preuves. Maxime de relation. Soyez pertinent. Maximes de manière 1. Evitez de vous exprimer de façon obscure. 2. 
Evitez l’ambiguité. 3. Soyez bref. 4. Soyez ordonné ». (Grice, 1975)

Le respect de ces maximes va orienter l'interprétation des énoncés lors de l'échange verbal. Le destinataire, supposant que son interlocuteur est coopératif, devra ajouter des informations au contenu littéral d'un énoncé pour comprendre ce que son interlocuteur communique. Grice dénomme ces informations supplémentaires des «implicitations » (en anglais « implicatures»). Dans l'exemple de la joute entre Beñat Iguaran et Oier Lakuntza le sens communiqué, conforme au but exigé par l'échange, est donc différent du sens littéral. Quand Oier Lakuntza énonce « ohea hurbil daukagu », «nous avons le lit à côté » il transgresse la maxime de manière. Il n'évite pas l'ambiguité, au contraire il la cultive. Pour faire disparaître la transgression de la maxime, le destinataire (Beñat Iguaran et le public) doit développer un certain nombre d'implicitations qui conduisent à l'énoncé. D'autre part, le bertsulari doit estimer le destinataire capable de développer son implicitation. Enfin, le destinataire devra supposer que le locuteur attend de lui qu'il développe cette implicitation. Il s'agit d'une implicature discursive, non conventionnelle ou lexicale qui elle à contrario serait non liée au contexte, non annulable par la suite du texte, mais détachable. "Nous avons le lit à côté » a pour implicature «nous pourrons éventuellement faire l'amour ». De sorte que l'implicature peut se définir comme ce qu'il faut ajouter pour rétablir l'observance de l'ensemble des maximes. C'est dans cet ajout, au moment même où les allocutaires développent cette implicitation (ils doivent penser que le lit n'est pas simplement un objet mais le lieu où la relation sexuelle a lieu et donc que si ils se fatiguent sur le sofa, ils auront l'opportunité de faire l'amour), que se situe le rire. Il réside dans ce qui est pensé à partir à la fois de ce qui est énoncé et de la situation où cela est dit. Le public a le choix entre plusieurs implicatures possibles. La première est de voir dans le lit un simple mobilier dans lequel on peut se reposer et dormir, la seconde retenue dans la joute par le public, le lieu d'une relation sexuelle. Seul le contexte, et en particulier l'énoncé de Beñat Iguaran, permet au public de choisir la seconde version plutôt que la première. Soit L1 le lit pour dormir et L2, le lit pour faire l'amour. Si le public préfère L2 à L1 c'est que le contexte le lui suggère. Le rire nait donc d'une préparation contextuelle. Il est en attente, en «stand by », potentiellement émancipable dès que Beñat Iguaran énonce «biok musuka hasi gintezken », «nous pourrions commencer à nous embrasser ». C'est l'énoncé de Beñat Iguaran qui oriente le public vers L2 plutôt que vers L1. Le rire peut donc se saisir comme l'aboutissement d'interactions entre une série d'ajouts successifs qui forment système. Chacun des éléments étant indispensables pour que le rire puisse être audible. Pour que L devienne risible, il doit obligatoirement être accompagné de (pré- $\mathrm{L}=(1)$ ), et de (post- $\mathrm{L}=3$ )) comme indiqué ci-dessous :

1) Mise en contexte («biok musuka hasi gintezken», «nous pourrions commencer à nous embrasser ») énoncé par Beñat Iguaran = pré-L

2) Enoncé de L par Oier Lakuntza ("Nous avons le lit à côté ») = L

3) Préférence du public pour L2 vis-à-vis de L1 par développement de l'implication = post-L

Ce n'est qu'à la condition: (pré-L)+(L)+(Post-L) que le rire est possible. D'où la formule suivante :

$$
(\text { pré-L })+(\mathrm{L})+((\text { Post-L, }(\mathrm{L} 2))=\text { RIRE }
$$


Dès lors que le sujet se prête au rire et qu'il est rendu possible par un contexte favorable, le rire est « pensable» (le contexte le rend possible). Le rire inhérent à la joute de l'improvisation orale improvisée est tributaire de conditions spécifiques.

1) Une ambiance générale qui prête au rire, l'improvisation doit s'émanciper dans une humeur enjouée. Plus cette condition sera remplie plus le rire pourra être libéré. En ce sens nous avons pu noter des différences notables entre les ambiances des concours, prix et championnat et celles des improvisations liées à des journées de fêtes. Lambiance festive étant généralement plus propice au rire, le public ayant plus le coeur à en rire que lors de journées consacrées aux championnats ou la tension des candidats est bien plus perceptible. La prédisposition au rire conditionne sa libération. Les concours restreignent l'envie d'en rire et les occasions d'en rire du fait même de l'enjeu. Mais le rire reste tout de même présent. Concours ou championnats n'interdisent pas le rire même si souvent ils le limitent.

2) Le rire apparaît d'autant plus facilement qu'il est attendu. Dans ces conditions le public est préparé à en rire, à l'avance, il y a alors corrélation entre intention de rendre comique de la part des improvisateurs et intention d'en rire de la part du public et des auditeurs. Le rire n'est plus à rechercher dans la profondeur de l'être, il est juste à révéler en surface. Si le public riait à la simple vue d'un improvisateur comme Mattin c'est que ce dernier l'avait déjà fait rire. Sa réputation le précédait. Ce souvenir d'avoir ri prépare le public à en rire de nouveau. Dans ces conditions le rire n'est plus induit par le dit, par l'énoncé à venir, il lui est antérieur, préexistant. La réputation de «faire rire » de l'improvisateur prédispose le public à « en rire » de nouveau. Le public en rit d'avance. La dépense du bertsulari pour faire rire le public sera proportionnelle à ses succès antérieurs. Plus il a fait rire et plus il sera pour lui facile de faire rire. Sa performance n'est pas isolée. Elle s'inscrit dans la diachronie, dans une chronologie qui informe autant que la prestation en elle-même. Linstant est conditionné par la mémoire collective, par l'histoire partagée et commune du public et de l'improvisateur.

3) Le rire répond au rire, c'est un plaisir répétitif. Il sera plus facile pour l'improvisateur de faire rire le public si ce dernier à déjà commencé à rire. Le rire est rarement isolé. Le rire se superpose au rire, il fonctionne sur le modèle de l'écho, de l'amplification, de la réactivation. A un effet ayant procuré « du plaisir d'en rire » vient s'en ajouter généralement un second, puis un troisième. La difficulté consiste à enclencher ou à déclencher le mécanisme. Le bertsulari se retrouve devant un choix multiple. Soit il est lui-même à l'origine du déclenchement originel. Dans ce cas il a la possibilité de réactiver ce processus qu'il a lui-même créé, en y revenant à sa guise une ou plusieurs fois. Soit il vient réutiliser un rire qui n'est pas le sien. Il use alors de l'effet réussi pour lequel le public a ri et qui est celui d'un autre bertsulari. Il imprime alors sa marque de fabrique en poursuivant le rire d'un autre qu'il fait devenir sien. Dans ce cas il n'a pas à aller chercher le rire bien loin puisqu'il est resté à la surface, et qu'il est encore tout frais dans la mémoire des auditeurs.

4) Pour faire rire, le bertsulari peut user de plusieurs stratagèmes qui prennent pour cible, soit sa propre personne (autodérision), soit son adversaire direct lors de la joute dialoguée, soit une tierce personne, un autre bertsulari présent lors de la performance, un autre improvisateur non présent le jour de la performance, un individu X, homme politique, responsable économique, acteur culturel, n'importe qui pourvu qu'il soit connu du public.

5) Pour qu'il puisse être efficace, le processus comique ne doit en rien dévoiler de sa technique d'élaboration. Il est indispensable que le « dessous des cartes » reste caché. Il ne supporte pas le surinvestissement opéré par l'attention. Si cette dernière est concentrée sur la 
comparaison dont le comique provient, elle atténue le processus. Comme ne note Freud : «Le processus de comparaison des dépenses doit impérativement rester automatique quand il est destiné à produire le plaisir comique ». (Freud, op cit, p. 386)

6) Le comique qui initie le rire est propre à chaque auditeur, à chaque spectateur de la performance. L'absolument comique n'est pas la règle. Chacun rit en fonction de son vécu personnel, en fonction de son âge, en fonction du degré de proximité qui le lie au contenu du comique, en fonction de sa «nature rieuse ». Certains sont plus enclins à en rire que d'autres. De plus, le rire peut parfois avoir ses destinataires privilégiés. Si c'est le cas, il ne fera rire que ceux qui se sentent directement concernés. Parfois ceux qui sont la cible des attaques ou des moqueries n'apprécient guère la plaisanterie. Moqueurs et moqués n'en rient pas de la même manière car il est parfois plus aisé de « rire de l'autre » que de « rire de soi-même ». Le rire généralisé ne s'émancipe que dans des cas d'exception. Le bertsulari fait travailler son imagination et trouve un énoncé qui le fait rire intérieurement mais qu'il se garde bien d'extérioriser. Il s'agit d'un rire intérieur inaudible. Le public percute et connecte, il rit, c'est le rire audible de l'auditoire. La formulation suivante est dès lors envisageable :

$$
\begin{gathered}
\mathrm{R}-\mathrm{C} \text { (contexte) }+\mathrm{R}-\mathrm{B} \text { (rire intérieur du bertsulari) }=\mathrm{R}-\mathrm{P} \text { (rire du public audible) } \\
\mathrm{R}-\mathrm{C}+\mathrm{R}-\mathrm{B}=\mathrm{R}-\mathrm{P}
\end{gathered}
$$

Analyser le rire, c'est donc focaliser son attention sur trois moments distincts mais inséparables. Le premier temps de l'étude du rire (R-C) est centré sur les conditions du rire (contexte de la joute mais aussi contexte social, économique, politique, culturel) qui conditionne les sujets risibles et trace les frontières du rire. Le second (R-B) est véritablement attaché au moment même où le bertsulari énonce ce qui fera rire, il est présent mais non encore audible, c'est le rire silencieux et enfin, le troisième (R-P), est celui de l'auditoire, perceptible à l'oreille, qui peut se définir par ce que l'on a coutume de nommer à proprement parler le rire.

Si l'étude du rire privilégie le mot entendu comme « résultante de l'association d'un sens donné à un ensemble de sons donnés susceptible d'un emploi grammatical donné » (Meillet, 1958 : 30), elle ne peut ignorer que le rire peut également résulter d'une manipulation d'unités plus ou moins grandes qui le dépassent. En deçà du mot, le rire peut en effet être initié par des unités plus petites comme les sons, les lettres ou les fragments de mots. Au-delà du mot, par des unités plus grandes comme les syntagmes ou les phrases. Le rire ne peut donc pas uniquement se définir comme un jeu sur les mots mais bien plus comme un jeu sur les sons, les lettres, les fragments, les syntagmes, les phrases et les mots, ce qui élargit considérablement son champ d'action. Lorsque le mot est préféré aux autres unités, le rire s'appuie sur ses trois aspects retenus par la définition de Meillet : sens, emploi grammatical et ensemble de sons.

Lanalyse des discours des bertsularis confirme que pour faire rire, ils recourent à une utilisation pléthorique de paronymes, d'homonymes, se jouent des sens, propres et figurés, des sonorités. Les improvisateurs basques jonglent avec les homophonies, les apophonies, les double sens, soit sans modifications, soit avec des transformations minimales. Le rire sémantique trouve sa source dans les manipulations des combinaisons syntagmatiques et paradigmatiques. Syntagmatiques dans lesquelles le mot peut entrer, des significations ou des emplois différents que ces combinaisons suscitent : elles participent de l'homonymie, de la polysémie, terme imputable à M. Bréal pour qualifier la multiplication des sens d'un même 
mot (Bréal, 1897 : 143-144). Paradigmatiques qui existent entre le mot et les mots qui peuvent formellement lui être substitués, antonymie, hyperonymie, hyponymie, synonymie. Lusage de cette dernière offre la possibilité aux bertsulari :

«(...) d’échanger des signifiés verbaux considérés comme équivalents, bien qu’ils soient configurés par des signifiants dissemblables; ou encore, celles de l'homonymie (des signifiants identiques par des signifiés sans relations) et de la polysémie (un même signifiant pour des signifiés apparentés). Bref, indissolublement liés dans la formulation du sens, formes et significations verbales n’en sont pas moins déliables par l'analyse. » (Tamba-Mecz, 1998 : 39)

Le langage des improvisateurs exprime les choses de façon incomplète et c'est au récepteur de compléter cette incomplétude. Pour que le message fasse rire, il requiert un consensus collectif sur le sens des mots. Le rire est subordonné à la double manipulation (du bertsulari, le locuteur, et du public, les allocutaires) de l'algèbre particulier propre à la langue basque. Il est initié par les échanges des signifiants et des signifiés, par les croisements des mots, les décompositions, les recompositions, les assemblages des uns avec les autres. Comme le note Henri Bergson : "On obtient un effet comique quand on affecte d'entendre une expression au propre, alors qu'elle était employée au figuré. » (Bergson : 88) Les homophonies internes ajoutent leur cohérence à celle des rimes, des homéotéleutes et autres assonances (homophonies de voyelles). La fluctuation des sonorités vient amplifier le jeu sur le sens et parfaire la résonance émotive et parfois risible d'un énoncé. Lapophonie qui introduit la modulation en passant d'un mot, d'un ensemble de syllabes, ou d'un nom à un autre, l'exemple du passage de Patxi à Paco est ici exemplaire, vient s'allier à l'homophonie pour armer le bertsulari dans sa quête $\mathrm{du}$ faire rire.

Mais la translation sémique et l'usage de double sens ne sauraient à eux seuls produire l'effet comique escompté. Elle est condition certes souvent nécessaire, mais non suffisante. Tout dépend alors du contexte et du sujet évoqué. L'exemple du passage de zakurrak à txakurrak est ici riche d'enseignement. Lorsqu'il s'agit de traiter d'une situation telle que le conflit politique au Pays basque, les limites du rire sont la plupart du temps atteintes et c'est cette délimitation qui explique que dans ce cas précis, l'usage du double sens ne devienne pas comique. L'exemple précédent lui est une excellente illustration de double sens accompagné de rire. Le basculement, le détournement, le « déplacement de la démarche de pensée »dirait Freud de Zazpi Etxe Frantzian en Zazpi etxe dauzka han permet d'accéder au rire parce que le contexte thématique dans lequel il s'émancipe (la moquerie envers l'auteur à succès Bernardo Atxaga) le lui permet. Comme le note Freud :

"Dans le cas du double sens, le mot d'esprit ne contient rien d'autre qu'un mot susceptible de donner lieu à une interprétation multiple et permettant à l'auditeur de passer d'une pensée à l'autre, passage qu'éventuellement - en forçant quelque peu les choses - on pourrait assimiler à un déplacement. En revanche, quand le mot d'esprit est fondé sur le déplacement, il contient lui-même une démarche de pensée dans laquelle un tel déplacement est accompli : le déplacement fait ici partie du travail qui a fabriqué le mot d'esprit, et non pas celui qui est nécessaire à sa compréhension. » (Freud, 1988: 118)

Le bertsularisme propose aux auditeurs un triple jeu. Sur les sonorités, sur le sens 
et sur l'emploi grammatical. Il prend comme support les mots, les lettres, les fragments, les syntagmes et les phrases. Tous ces exemples nous renvoient aux différentes catégories définies par Sigmund Freud dans sa «technique du mot d'esprit ». On y retrouve en effet l'utilisation du même matériel (tout et parties, modification de l'ordre des mots, légère modification, même mots pris au sens plein et au sens vide), le double sens (nom et signification concrète, signification métaphorique et signification concrète, double sens proprement dit, jeu de mots, équivoque, double sens accompagné d'une allusion). Mais plus difficile est à repéré la condensation (accompagnée de la formation d'un mot mixte, accompagnée d'une modification).

Le rire des Basques est donc repérable à un triple jeu :

1) sur les techniques de composition (figures rhétoriques, métaphores, amphibologie, équivoque, double sens, ironie, allusions, insinuations...)

2) sur les relations structurelles syntagmatiques (homonymie, polysémie) et paradigmatiques (synonymie, hyponymie, antonymie, hyperonymie)

3) sur ce qui est dit (mots, syntagmes, phrases, sons...)

Le rire s'il se joue de la langue et de ses spécificités lexico-grammaticales pour se faire audible s'inspire dans le même temps de faits historico-socioculturels qui l'orientent. Ces faits le chargent de sens et lui impriment son originalité. C'est la spécificité du contenu des discours qui explique que l'on ne rit pas de la même chose partout. C'est en cela que le rire des Basques diffère de celui des Anglais, des Français, des Espagnols, des Catalans, des Américains, des Allemands, des Chinois, des Japonais ou des Inuits. Cependant, même s'il semble acquis que les Basques partagent avec d'autres certaines thématiques, c'est la manière de les traiter, subordonnée à l'organisation de la langue avec ses catégories ontologiques, ses relations structurelles, son vocabulaire, sa syntaxe, ses règles, ses lois et sa grammaire, qui donne au rire des Basques sa singularité. Ce qui est dit et la manière dont ce qui est dit est dit ont partie liée. Le rire sémantique des Basques est subordonné et conditionné, à la fois à la structure de la langue (euskara), aux rapports des signes à leurs utilisateurs et en même temps, au contexte historico-politico-socio-culturel du Pays Basque (Euskal Herria), au sein desquels il s'émancipe. Il appartient au rirologue d'en saisir la diversité, la spécificité et la cohérence globale. Il est aidé dans sa quête cognitive, par les disciplines complémentaires que sont l'ethnologie, l'histoire, la politologie, la sémantique, la lexicologie, la pragmatique et la linguistique, qui lui permettent de rendre compte le plus fidèlement possible de son ubiquité. Cette approche pluri-trans- disciplinaire semble en effet la seule à permettre au chercheur d'appréhender les différents niveaux d'analyse (du vocable au texte ou du lexique au discours) qui constituent, avec sa variabilité qui complexifie sa délimitation, le propre du rire.

L'ouvre bertsularistique est démiurgique, elle crée par des manipulations de mots, de syntagmes, par des poèmes nommés bertsu une terre nouvelle, un Pays Basque nouveau. Elle est doublement créatrice, d'une part de la psyché collective, d'autre part d'un milieu social qu'elle informe. C'est l'œuvre qui crée la sensibilité d'un groupe « contrairement à l'hypothèse réductrice inverse ». (Durand, 1990 : 391) La tentation formaliste pour qui rimes et assonances dépasseraient le sens est subordonnée à l'impérialisme du message. La forme du dit ne dépasse jamais le dit. Phonétique, grammaire et lexique sont mis à contribution pour dire le nouveau monde. L'histoire version bertsulari émerge des petits faits de l'histoire évènementielle. Les improvisateurs exhument les mots des dictionnaires, réchauffent les syntaxes de la froideur des grammaires, transcendent les données du monde réel. Le passage dans l'imaginaire permet la transformation des lieux, des lexiques, des personnages. Sur les traces des nouveaux sujets 
actants, nait un ailleurs, un sur-monde destiné à remplacer l'ici et le maintenant, hic et nunc, jugé inacceptable. Par la voix du bertsulari, le fantasme prend corps. Il devient réalité, univers exemplaire qui dicte sa morale. Ce ne sont plus les protagonistes de la fiction bertsularistique qui deviennent les héros, mais bien ceux par qui le dire et le rire adviennent.

La production bertsularistique érige un Pays Basque légendaire, un espace où vit un peuple qui chante des bertsu improvisés. Elle vient s'adosser à la production littéraire générale dessinant un pays où les habitants dansent, chantent et jouent à la pelote, à une presse qui se fait l'écho d'un terrorisme non désactivé. Les bertsu inventent les paysages de l'âme basque. Les sujets imposés sécrètent une multitude de décors et de personnages. Ils comblent le besoin de changement des improvisateurs. De la dialectique étroite entre imaginaire et réalité nait le rire. Le bertsulari est un agoradorateur adoré par l'agora. La foule est sa raison d'être. Une pièce de théâtre de mime est universellement exportable et elle l'est d'autant mieux que ce qui est énoncé est minime voire inexistant. Le geste s'exporte mieux que la parole. Il suffit pour s'en persuader d'observer un locuteur qui ne maîtrise pas la langue vernaculaire, tenté de se faire comprendre pour s'en persuader. Mais dès que le geste se fait plus discret ou s'efface, la langue règne. Dès lors, toute exportation ou importation devient beaucoup plus difficile. Le bertsularisme fait partie de ces pratiques culturelles non exportables en terre non bascophone. C'est la raison pour laquelle toute traduction paraît si périlleuse parce qu'elle a tendance à perdre en chemin une part de l'essence originelle. C'est certainement aussi cela qui explique la faible bibliographie concernant le sujet ici traité.

Visionnaires de l'indépendance du Pays Basque, les bertsulari en sont les constructeurs, tout autant si ce n'est plus, que tous les partis politiques indépendantistes réunis. Les prémisses d'un Pays Basque pacifié seront audibles dans la bouche des improvisateurs, bien avant que dans celle des politiques. Une paix qui s'annoncera sur des pattes de colombes, pour reprendre la formule de Nietzsche, comme tous les grands évènements. Car ils sont ceux par qui, ce qui adviendra, est déjà présent. Prédicateurs de l'avenir, ils nous chantent ce que le futur sera (où tout du moins ce qu'ils voudraient qu'il soit). Utopiques, ils surpassent les politiques dans la mesure où ils transmutent en réalité l'imaginaire contenu dans l'idéologie. Acteurs de la transformation sociétale, ils sculptent le réel à leur image. Ils tendent à la société basque, le miroir de ce qu'ils voudraient qu'elle soit, tout autant que ce qu'elle voudrait bien être. Le bertsularisme échappe pour sa part à toute tentative de folklorisation. Ici ni danse, ni beaux costumes, ni gestuelle exacerbée à observer pour ceux qui viennent côtoyer les improvisateurs. A la manière du maître du Bunraku japonais, le bertsulari a la tête « découverte, nu, sans fard, ce qui lui confère un cachet civil (non théâtral), son visage est offert à la lecture des spectateurs». (Barthes, 1968- 1971, p. 36) Si certains observateurs des mascarades en Soule ont vu en ce carnaval populaire un spectacle de danse c'est qu'ils ne maîtrisaient pas la langue, se contentant de voir dans cette fête un spectacle de danse parsemé de jeux (Dicharry, 2012). Ici, une telle méprise est impossible. Les curieux non bascophones se contenteront de la beauté du chant, de la danse, de la sculpture ou de la peinture pour satisfaire leur soif d'esthétique basque.

\section{Bibliographie}

Armengaud, F. 1998. La pragmatique. Paris: PUF.

Barthes, R. 1985. L'aventure sémiologique, Paris, Editions du Seuil.

Barthes, R. 1968. Textes 1968, L'effet de réel. In CEuvres complètes, III, Livres, Textes, Entretiens, 
1968-1971, Paris, Seuil, 2002. 25 - 52.

Bergson, H.1940 [2002]. Le rire : essai sur la signification du comique. Paris: PUF, Quadrige.

Dicharry, E. 2013. L'écologie de l'éducation. Un anthropologue à l'école du bertsularisme, Paris: l'Harmattan.

Dicharry, E. 2013. Le rire des Basques, Paris: L'Harmattan.

Dicharry, E. 2012. Du rite au rire. Le discours des mascarades souletines, Paris: LHarmattan.

Dicharry, E. 2012. L'humour et l'absurde. Une étude de cas : l'oeuvre d'Esther Ferrer. RIEV (Revista International de Estudios Bascos), 57, 2: 312-345.

Dicharry, E. 2011. Bertsolaritza eta umorea. Bertsolari aldizkaria, 82. 42- 83.

Dicharry, E. 2010. Pour une anthropologie de la fête au Pays Basque. Lapurdum XIV. 33-53.

Dicharry, E. 2009. De l'expérience du terrain aux théories ethnolinguistiques. Lankidetza 51, 461-470.

Dicharry, E. 1999. Approche ethnolinguistique des mascarades souletines. Oihenart, 16. 87 136.

Durand, G. 1990. Les fondements de la création littéraire. In Encyclopaedia Universalis, 391400.

Freud, S. 1988. Le mot d'esprit et sa relation à l'inconscient, (trad française), Paris: Gallimard.

Garzia J. 2007. History of Improvised Bertsolaritza : A Proposal. Oral Tradition, 22,2. 77-115.

Garzia J. 2005. A Theoretical Framework for Improvised Bertsolaritza. In Voicing the moment : improvised oral poetry and Basque tradition, Center for Basque Studies, Conference Papers Series: University of Reno.

Garzia J. 2000. Testua eta ingurua. In Gaur egungo bertsolarien baliabide erretorikopoetikoak. Bilbao: EHU Press. 208-275.

Grice, H.P. 1975. Logic and conversation. In Cole, P. \& J. Morgan (eds), Syntax and semantics. Speech acts, Vol. 3, New York: Academic Press. 41-58.

Lacan, J. 1998. Le séminaire, Livre V, Les formations de l'inconscient. Paris: Seuil.

Martinet, A. 1960 [1964]. Eléments de linguistique générale, Paris, Colin.

Meillet, A. 1958. Linguistique historique et linguistique générale, Paris: Champion.

Montaigne, Essais, III.

Peirce, Ch.S. 1978. Écrits sur le signe (rassemblés, traduits et commentés par G. Deledalle), Paris: Seuil.

Riffaterre, M. 1982. Lillusion référentielle. In Littérature et réalité, Paris: Editions du Seuil, 91118. [Reprise de l'article publié dans Columbia Review, 57, 2, 1978].

Saussure, F. de. 1913 [1995]. Cours de Linguistique Générale, Paris: Payot.

Tamba-Mecz, I. 1988 [1998]. La sémantique, Paris: PUF.

Wittgenstein, L. 1980. Grammaire philosophique. éd. R. Rhees, trad, M.-A Lescourret, Paris: Gallimard.

Zumthor, P. 1983. Introduction à la poésie orale. Paris: Seuil. 
